\title{
Determination of the Equilibrium Constant and Rate Constant of Protein-Oligonucleotide Complex Dissociation under the Conditions of Ideal-Filter Capillary Electrophoresis
}

\author{
An T. H. Le, Svetlana M. Krylova, , Sergey N. Krylov* \\ Department of Chemistry and Centre for Research on Biomolecular Interactions, \\ York University, Toronto, Ontario M3J 1P3, Canada
}

$\underline{\text { Supporting Information }}$

Note S1: List of symbols S2

Note S2: Calculating $\boldsymbol{R}$ from the velocity-corrected peak areas via double-passage approach

Note S3: Calculating $k_{\text {off }}$ by varying $E$ during the second passage ........................................S3

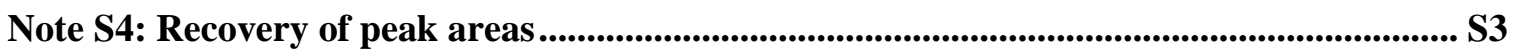

Figure S1. Double-passage experiment performed with $1 \mu \mathrm{M}$ GFP in IFCE run buffer............ S3

Table S1. Recovery of peak areas for double-passage experiment with $1 \mu \mathrm{M}$ GFP ................. S4

Note S5: Recovery of $R$................................................................................................................ S4

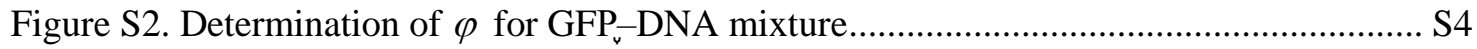

Figure S3. Double-passage experiments of GFP-DNA mixtures with [DNA]/[GFP] ratios

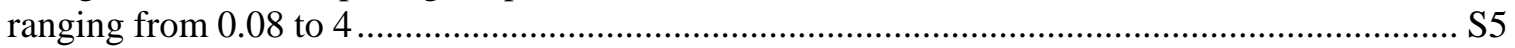

Table S2. Recovery of $R$ for double-passage experiment of GFP-DNA mixtures. ................. S6

Note S6: Experimental determination of $K_{\mathrm{d}}$ and $\boldsymbol{k}_{\text {off }}$ of protein-aptamer complex ............... S7

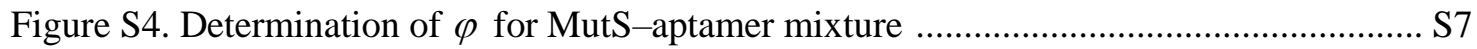

Figure S5. Double-passage experiment with mixture of MutS protein and its aptamer under

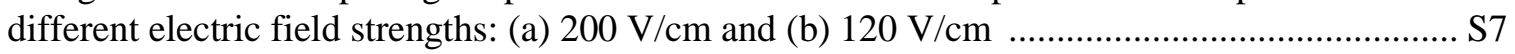

Table S3. Experimental determination of $K_{\mathrm{d}}$ of MutS-aptamer complex .............................. S7

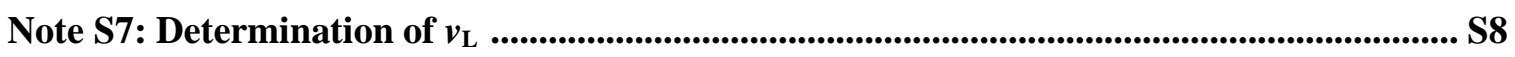

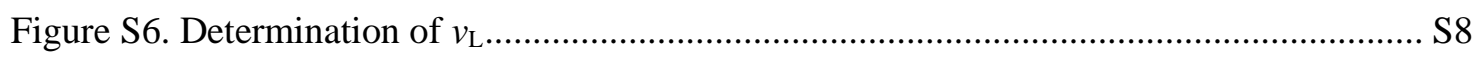

For more information see also the Excel file in the Supporting Information. 


\section{Note S1: List of symbols}

a coefficient of proportionality between the peak area and the concentration of the fluorescent oligonucleotide

$A_{1} \quad$ area of the first pressure-driven peak

$A_{2} \quad$ area of the second electrophoretic peak

$d \quad$ the distance from the capillary inlet to the center of the detection window

$d_{1} \quad$ distance between the center of the stopped plug and the center of the detection window

$D \quad$ diffusion coefficient of the largest-size analyte

E vector of the electric field

E magnitude of $\mathbf{E}$

EM equilibrium mixture

EOF electroosmotic flow

$k_{\text {off }} \quad$ rate constant of complex dissociation

$k_{\text {on }} \quad$ rate constant of complex formation

$K_{\mathrm{d}} \quad$ equilibrium dissociation constant

$l \quad$ length of sample plug

$[\mathrm{L}]_{0} \quad$ initial concentration of ligand

$[\mathrm{L}]_{\mathrm{eq}} \quad$ equilibrium concentration of ligand

$\boldsymbol{\mu}_{\mathrm{EOF}} \quad$ electrophoretic mobility vector of electroosmotic flow

$\boldsymbol{\mu}_{\mathrm{L}} \quad$ electrophoretic mobility vector of ligand

$\mu_{\mathrm{L}} \quad$ electrophoretic mobility of ligand

$\mu_{\mathrm{PL}} \quad$ electrophoretic mobility vector of complex

$\mu_{\mathrm{PL}} \quad$ electrophoretic mobility of complex

$[\mathrm{P}]_{0} \quad$ initial concentration of protein

$[\mathrm{P}]_{\text {eq }} \quad$ equilibrium concentration of protein

$[\mathrm{PL}]_{\mathrm{eq}}$ equilibrium concentration of complex

$r \quad$ capillary radius

$\mathrm{R} \quad$ fraction of unbound ligand at equilibrium

$t_{1} \quad$ the time span from the beginning of EM plug propagation to the time when the plug's center reaches the center of the detection window

$t_{2} \quad$ time span from the plug center's passing the center of detection window to the end of plug propagation

$t_{3} \quad$ time span from the beginning of electrophoresis to the moment when the center of the plug of PL reaches the center of the detection window

$t_{3}$, varied value of $t_{3}$ by changing the electric field strength

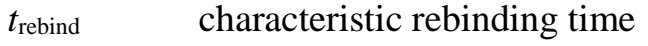

$t_{\text {sep }}$

$v_{\mathrm{av}}$

$\mathbf{V}_{\mathrm{L}}$

$v_{\mathrm{L}}$

$\mathbf{V}_{P}$

VPL

$v_{\mathrm{PL}}$

characteristic separation time of complex from unbound ligand and protein

pressure-driven velocity during the first passage

electrophoretic velocity vector of ligand

electrophoretic velocity of ligand

electrophoretic velocity vector of protein

electrophoretic velocity vector of complex

electrophoretic velocity of complex during the second passage

$\varphi \quad$ relative quantum yield of a fluorescent label within the protein-oligonucleotide complex with respect to that of the free oligonucleotide 
Note S2: Calculating $\boldsymbol{R}$ from the velocity-corrected peak areas via double-passage approach

$$
\begin{array}{r}
R=\frac{[\mathrm{L}]_{\mathrm{eq}}}{[\mathrm{L}]_{0}}=\frac{[\mathrm{L}]_{\mathrm{eq}}}{[\mathrm{PL}]_{\mathrm{eq}}+[\mathrm{L}]_{\mathrm{eq}}}=\frac{\left(\varphi[\mathrm{PL}]_{\mathrm{eq}}+[\mathrm{L}]_{\mathrm{eq}}\right)-\varphi[\mathrm{PL}]_{\mathrm{eq}}}{\left\{\varphi[\mathrm{PL}]_{\mathrm{eq}} / \varphi\right\}+\left\{\left(\varphi[\mathrm{PL}]_{\mathrm{eq}}+[\mathrm{L}]_{\mathrm{eq}}\right)-\varphi[\mathrm{PL}]_{\mathrm{eq}}\right\}}= \\
\frac{A_{1} v_{\mathrm{av}} / a-A_{2} v_{\mathrm{PL}} / a}{\left\{\left(A_{2} v_{\mathrm{PL}} / a\right) / \varphi\right\}+\left\{A_{1} v_{\mathrm{av}} / a-A_{2} v_{\mathrm{PL}} / a\right\}}=\frac{A_{1} v_{\mathrm{av}}-A_{2} v_{\mathrm{PL}}}{A_{2} v_{\mathrm{PL}} / \varphi+A_{1} v_{\mathrm{av}}-A_{2} v_{\mathrm{PL}}}
\end{array}
$$

\section{Note S3: Calculating $\boldsymbol{k}_{\text {off }}$ by varying $E$ during the second passage}

The value of $k_{\text {off }}$ can be determined by using varying $t_{3}$ via changing the electric field strength $\left(E^{\prime}<E\right)$. A two-point approach (with two different values of $\left.t_{3}: t_{3}<t_{3}{ }^{\prime}\right)$ can be used for a simple assessment of $k_{\text {off: }}$

$$
\begin{aligned}
& 1-R\left(t_{3}\right)=\left(1-R_{t=0}\right) e^{-k_{\mathrm{off}} t_{3}}, \quad 1-R\left(t_{3}{ }^{\prime}\right)=\left(1-R_{t=0}\right) e^{-k_{\mathrm{off}} t_{3}{ }^{\prime}} \\
& \frac{1-R\left(t_{3}\right)}{1-R\left(t_{3}\right)}=e^{-k_{\mathrm{off}}\left(t_{3}{ }^{\prime}-t_{3}\right)}, \quad \ln \left(\frac{1-R\left(t_{3}{ }^{\prime}\right)}{1-R\left(t_{3}\right)}\right)=-k_{\mathrm{off}}\left(t_{3}{ }^{\prime}-t_{3}\right) \\
& k_{\mathrm{off}}=\frac{\ln \left(\frac{1-R\left(t_{3}\right)}{1-R\left(t_{3}{ }^{\prime}\right)}\right)}{t_{3}{ }^{\prime}-t_{3}}
\end{aligned}
$$

\section{Note S4: Recovery of peak areas}

In order to evaluate the recovery of velocity-corrected peak areas via the double-passage approach, we used $1 \mu \mathrm{M}$ GFP as an analyte mimicking PL in terms of electrophoretic mobility. The experiment was repeated 10 times using the default conditions: $d=10.2 \mathrm{~cm}, r=37.5 \mu \mathrm{m}$, $l=1.09 \mathrm{~cm}$, pressure equal to $0.30 \mathrm{psi}$, total capillary length $=50 \mathrm{~cm}, E=200 \mathrm{~V} / \mathrm{cm}$ (Figure S1). Detailed calculation for recovery of peaks areas can be found from Table S1.

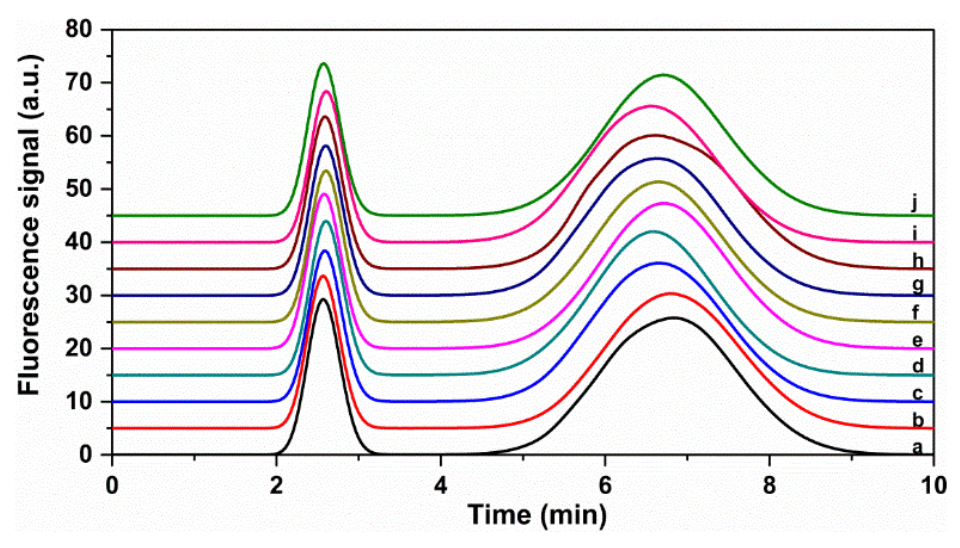

Figure S1. Double-passage experiment performed with $1 \mu \mathrm{M}$ GFP in IFCE running buffer $(50 \mathrm{mM}$ Tris- $\mathrm{HCl} \mathrm{pH} \mathrm{7.0,100} \mathrm{mM} \mathrm{NaCl})$. Ten repetitions were done to determine the average velocity-corrected areas and to assess the error. 
Table 1. Recovery of peak areas for double-passage experiment with $1 \mu \mathrm{M}$ GFP

\begin{tabular}{|c|c|c|c|c|c|c|c|c|c|}
\hline Figure & $A_{1}$ & $A_{2}$ & $\begin{array}{c}t_{1} \\
\min \end{array}$ & $\begin{array}{c}v_{\text {av }} \\
\mathrm{cm} / \mathrm{min}\end{array}$ & $\begin{array}{c}t_{2} \\
\min \end{array}$ & $\begin{array}{l}d_{1} \\
\mathrm{~cm}\end{array}$ & $\begin{array}{c}t_{3} \\
\min \end{array}$ & $\begin{array}{c}v_{\mathrm{PL}} \\
\mathrm{cm} / \mathrm{min}\end{array}$ & $\left(A_{1} v_{\mathrm{av}}\right) /\left(A_{2} v_{\mathrm{PL}}\right)$ \\
\hline $\begin{array}{l}\text { S1, } \\
\text { trace a }\end{array}$ & $8.70 \times 10^{7}$ & $3.11 \times 10^{8}$ & 2.85 & 3.39 & 0.933 & 3.16 & 3.36 & 0.941 & 1.01 \\
\hline $\begin{array}{c}\text { S1, } \\
\text { trace b }\end{array}$ & $8.43 \times 10^{7}$ & $2.91 \times 10^{8}$ & 2.85 & 3.39 & 0.933 & 3.16 & 3.28 & 0.963 & 1.02 \\
\hline $\begin{array}{l}\text { S1, } \\
\text { trace c }\end{array}$ & $8.44 \times 10^{7}$ & $2.92 \times 10^{8}$ & 2.87 & 3.36 & 0.913 & 3.07 & 3.16 & 0.971 & 1.00 \\
\hline $\begin{array}{l}\text { S1, } \\
\text { trace d }\end{array}$ & $8.60 \times 10^{7}$ & $2.92 \times 10^{8}$ & 2.88 & 3.35 & 0.900 & 3.01 & 3.11 & 0.969 & 1.02 \\
\hline $\begin{array}{c}\text { S1, } \\
\text { trace e }\end{array}$ & $8.65 \times 10^{7}$ & $2.98 \times 10^{8}$ & 2.86 & 3.37 & 0.921 & 3.11 & 3.20 & 0.972 & 1.01 \\
\hline $\begin{array}{c}\text { S1, } \\
\text { trace f }\end{array}$ & $8.41 \times 10^{7}$ & $2.92 \times 10^{8}$ & 2.88 & 3.35 & 0.900 & 3.01 & 3.14 & 0.959 & 1.01 \\
\hline $\begin{array}{l}\text { S1, } \\
\text { trace g }\end{array}$ & $8.38 \times 10^{7}$ & $2.95 \times 10^{8}$ & 2.88 & 3.35 & 0.900 & 3.01 & 3.12 & 0.967 & 0.98 \\
\hline $\begin{array}{l}\text { S1, } \\
\text { trace h }\end{array}$ & $8.48 \times 10^{7}$ & $2.89 \times 10^{8}$ & 2.88 & 3.36 & 0.908 & 3.05 & 3.11 & 0.981 & 1.00 \\
\hline $\begin{array}{l}\text { S1, } \\
\text { trace i }\end{array}$ & $8.41 \times 10^{7}$ & $2.84 \times 10^{8}$ & 2.89 & 3.34 & 0.896 & 3.00 & 3.05 & 0.984 & 1.01 \\
\hline $\begin{array}{c}\text { S1, } \\
\text { trace j }\end{array}$ & $8.40 \times 10^{7}$ & $2.92 \times 10^{8}$ & 2.85 & 3.38 & 0.929 & 3.14 & 3.21 & 0.980 & 0.99 \\
\hline
\end{tabular}

See the accompanying Excel file for full data and error calculations

\section{Note S5: Recovery of $R$}
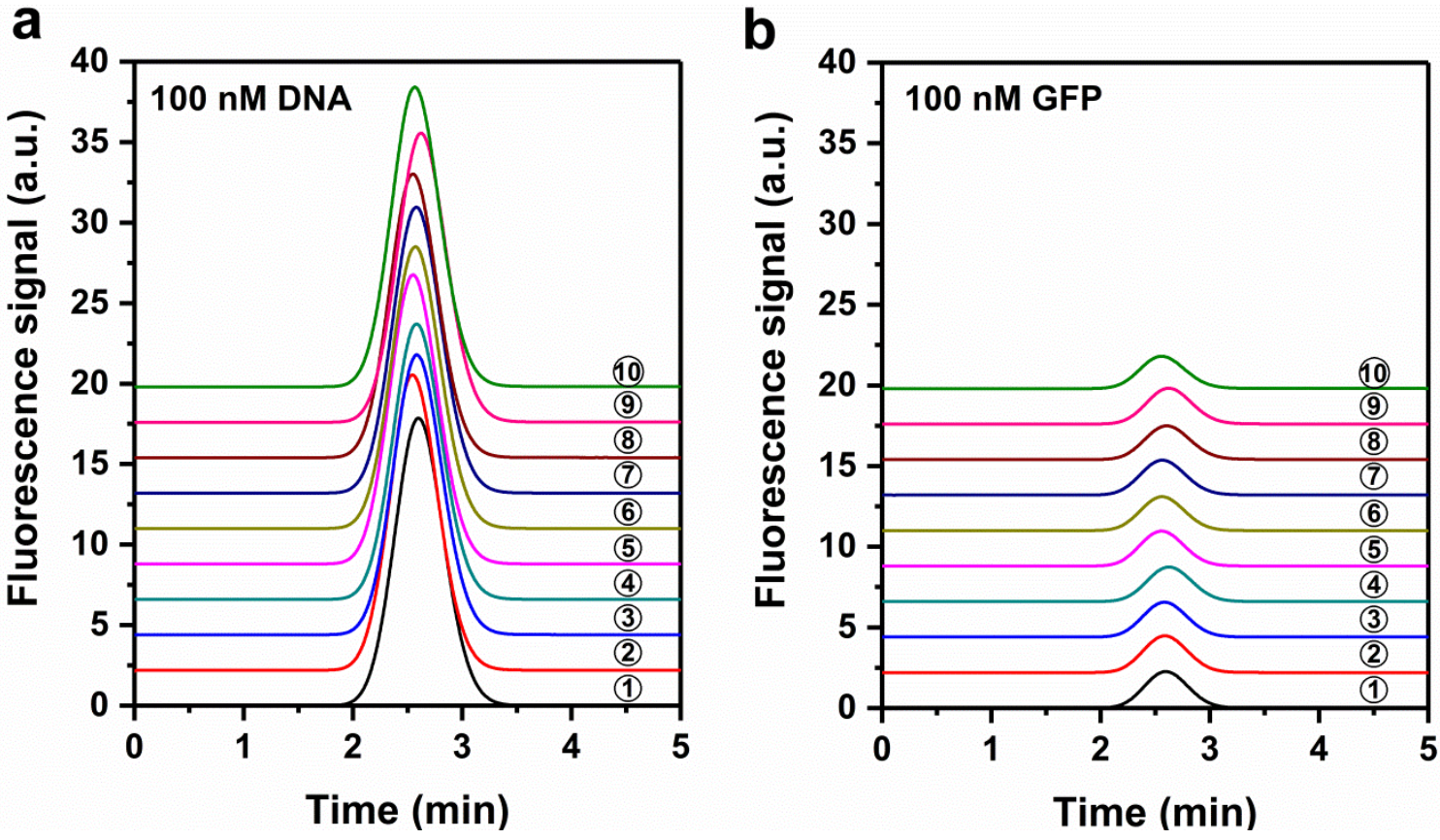

Figure S2. Determination of $\varphi$ for GFP-DNA mixture. Areas of peaks of GFP and DNA yield a ratio of $\approx 0.11$. Each set of experiments was performed 10 times. See the accompanying Excel file for full data and error calculations. 


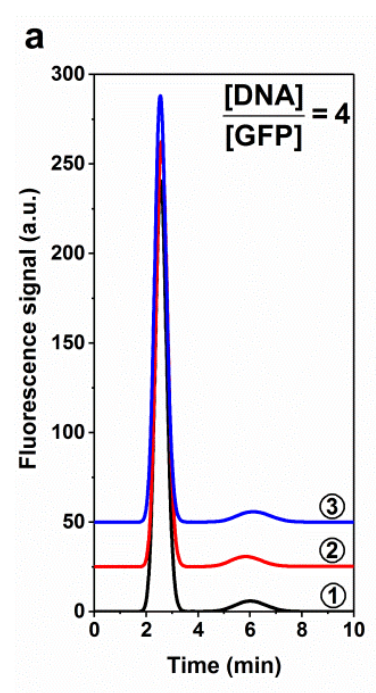

e

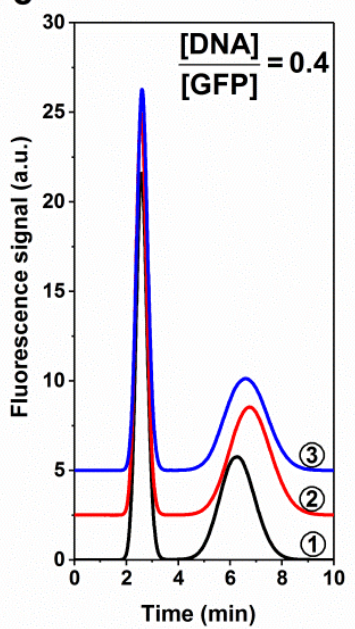

b

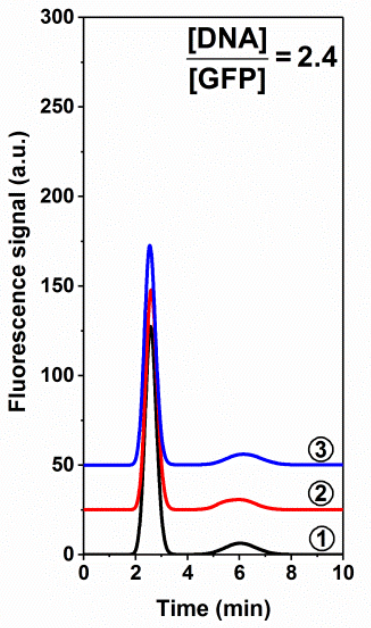

$f$

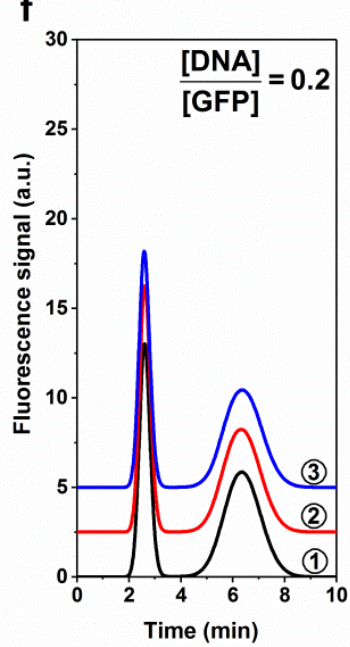

d
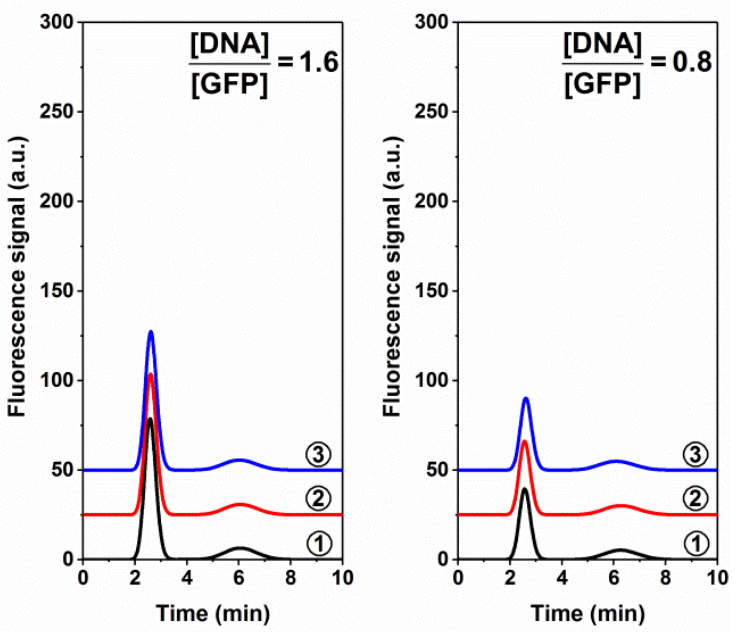

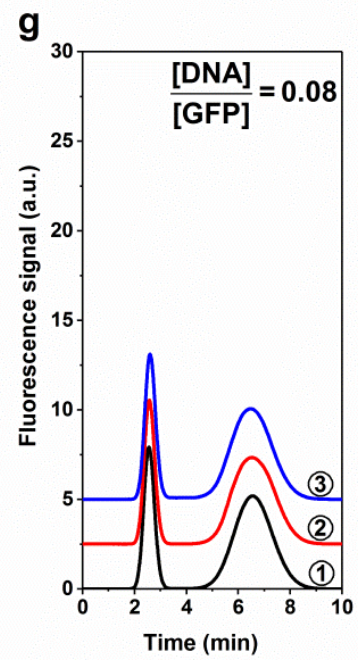

Figure S3. Double-passage experiments of GFP-DNA mixtures with [DNA]/[GFP] ratios ranging from 0.08 to 4 . Each set of experiments was performed in triplicates. 
Table S2. Recovery of $R$ for double-passage experiment of GFP-DNA mixtures.

\begin{tabular}{|c|c|c|c|c|c|c|c|c|c|c|c|}
\hline$\frac{[\mathrm{DNA}]}{[\mathrm{GFP}]}$ & Figure & $A_{1}$ & $A_{2}$ & $\begin{array}{c}t_{1} \\
\min \end{array}$ & $\begin{array}{c}v_{\mathrm{av}} \\
\mathrm{cm} / \mathrm{min}\end{array}$ & $\begin{array}{c}t_{2} \\
\min \end{array}$ & $\begin{array}{l}d_{1} \\
\mathrm{~cm}\end{array}$ & $\begin{array}{c}t_{3} \\
\text { min }\end{array}$ & $\begin{array}{c}v_{\mathrm{PL}} \\
\mathrm{cm} / \mathrm{min}\end{array}$ & $R$ & Mean $R \pm \mathrm{SD}$ \\
\hline \multirow{3}{*}{4} & S3-a, 1 & $7.88 \times 10^{8}$ & $5.18 \times 10^{7}$ & 2.85 & 3.39 & 0.937 & 3.18 & 2.52 & 1.26 & 0.814 & \multirow{3}{*}{$0.813 \pm 0.003$} \\
\hline & S3-a, 2 & $7.84 \times 10^{8}$ & $4.93 \times 10^{7}$ & 2.88 & 3.36 & 0.908 & 3.05 & 2.34 & 1.30 & 0.815 & \\
\hline & S3-a, 3 & $7.79 \times 10^{8}$ & $5.44 \times 10^{7}$ & 2.83 & 3.41 & 0.954 & 3.26 & 2.64 & 1.23 & 0.810 & \\
\hline \multirow{3}{*}{2.4} & S3-b, 1 & $4.15 \times 10^{8}$ & $5.70 \times 10^{7}$ & 2.86 & 3.37 & 0.921 & 3.11 & 2.54 & 1.22 & 0.677 & \multirow{3}{*}{$0.672 \pm 0.005$} \\
\hline & S3-b, 2 & $4.00 \times 10^{8}$ & $5.65 \times 10^{7}$ & 2.89 & 3.34 & 0.896 & 3.00 & 2.43 & 1.23 & 0.667 & \\
\hline & S3-b, 3 & $3.93 \times 10^{8}$ & $5.59 \times 10^{7}$ & 2.83 & 3.41 & 0.950 & 3.24 & 2.64 & 1.23 & 0.671 & \\
\hline \multirow{3}{*}{1.6} & S3-c, 1 & $2.56 \times 10^{8}$ & $5.15 \times 10^{7}$ & 2.87 & 3.37 & 0.917 & 3.09 & 2.55 & 1.21 & 0.585 & \multirow{3}{*}{$0.582 \pm 0.003$} \\
\hline & S3-c, 2 & $2.56 \times 10^{8}$ & $5.40 \times 10^{7}$ & 2.88 & 3.35 & 0.900 & 3.01 & 2.58 & 1.17 & 0.580 & \\
\hline & S3-c, 3 & $2.51 \times 10^{8}$ & $5.23 \times 10^{7}$ & 2.90 & 3.34 & 0.888 & 2.96 & 2.52 & 1.18 & 0.581 & \\
\hline \multirow{3}{*}{0.8} & S3-d, 1 & $1.27 \times 10^{8}$ & $5.13 \times 10^{7}$ & 2.85 & 3.39 & 0.933 & 3.16 & 2.76 & 1.15 & 0.411 & \multirow{3}{*}{$0.423 \pm 0.011$} \\
\hline & S3-d, 2 & $1.33 \times 10^{8}$ & $5.13 \times 10^{7}$ & 2.85 & 3.39 & 0.937 & 3.18 & 2.79 & 1.14 & 0.424 & \\
\hline & S3-d, 3 & $1.30 \times 10^{8}$ & $4.84 \times 10^{7}$ & 2.90 & 3.34 & 0.888 & 2.96 & 2.62 & 1.13 & 0.433 & \\
\hline \multirow{3}{*}{0.4} & S3-e, 1 & $6.90 \times 10^{7}$ & $5.80 \times 10^{7}$ & 2.86 & 3.37 & 0.921 & 3.11 & 2.78 & 1.12 & 0.222 & \multirow{3}{*}{$0.225 \pm 0.013$} \\
\hline & S3-e, 2 & $7.00 \times 10^{7}$ & $7.03 \times 10^{7}$ & 2.85 & 3.38 & 0.929 & 3.14 & 3.23 & 0.97 & 0.213 & \\
\hline & S3-e, 3 & $6.71 \times 10^{7}$ & $5.97 \times 10^{7}$ & 2.89 & 3.34 & 0.896 & 3.00 & 3.08 & 0.97 & 0.240 & \\
\hline \multirow{3}{*}{0.2} & S3-f, 1 & $4.06 \times 10^{7}$ & $6.12 \times 10^{7}$ & 2.88 & 3.35 & 0.900 & 3.01 & 2.85 & 1.06 & 0.108 & \multirow{3}{*}{$0.114 \pm 0.005$} \\
\hline & S3-f, 2 & $4.10 \times 10^{7}$ & $5.69 \times 10^{7}$ & 2.86 & 3.37 & 0.919 & 3.10 & 2.83 & 1.10 & 0.118 & \\
\hline & S3-f, 3 & $4.08 \times 10^{7}$ & $5.85 \times 10^{7}$ & 2.87 & 3.36 & 0.913 & 3.07 & 2.87 & 1.07 & 0.116 & \\
\hline \multirow{3}{*}{0.08} & S3-g, 1 & $2.41 \times 10^{7}$ & $5.64 \times 10^{7}$ & 2.84 & 3.40 & 0.942 & 3.20 & 3.05 & 1.05 & 0.041 & \multirow{3}{*}{$0.042 \pm 0.001$} \\
\hline & S3-g, 2 & $2.43 \times 10^{7}$ & $5.73 \times 10^{7}$ & 2.86 & 3.38 & 0.925 & 3.12 & 3.03 & 1.03 & 0.041 & \\
\hline & S3-g, 3 & $2.48 \times 10^{7}$ & $5.85 \times 10^{7}$ & 2.88 & 3.35 & 0.900 & 3.01 & 2.99 & 1.01 & 0.043 & \\
\hline
\end{tabular}


Note S6: Experimental determination of $K_{\mathrm{d}}$ and $\boldsymbol{k}_{\text {off }}$ of protein-aptamer complex
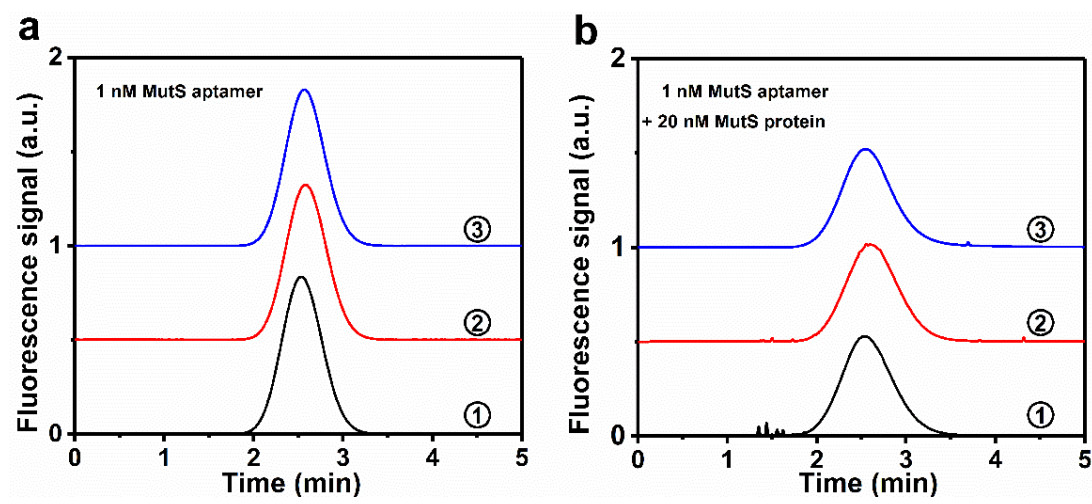

Figure S4. Determination of $\varphi$ for MutS-aptamer mixture. Areas of peaks PL and L, at full complex saturation and at absence of target protein yield a ratio of $\approx 0.80$. Each set of experiments was performed in triplicates. See the accompanying Excel file for full data and error calculations.

a

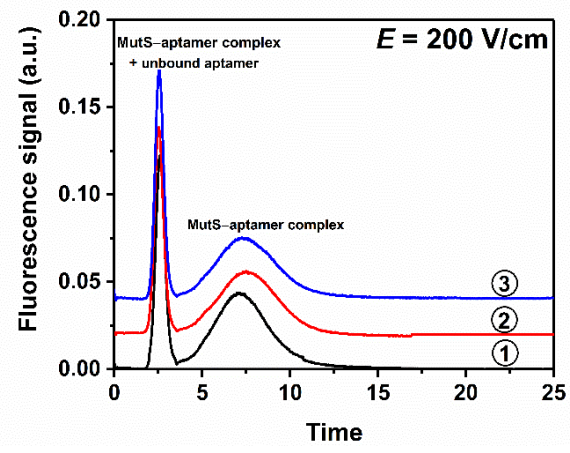

b

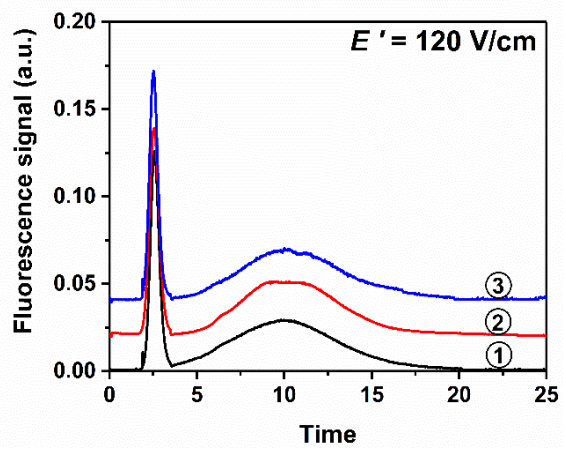

Figure S5. Double-passage experiment with mixture of MutS protein and its aptamer under different electric field strengths: (a) $200 \mathrm{~V} / \mathrm{cm}$ and (b) $120 \mathrm{~V} / \mathrm{cm}$. Each set of experiments was performed in triplicates. The sample mixture contained $0.50 \mathrm{nM}$ MutS and $0.20 \mathrm{nM}$ aptamer.

Table S3. Experimental determination of $K_{\mathrm{d}}$ of MutS-aptamer complex

\begin{tabular}{|c|c|c|c|c|c|c|c|c|c|c|c|c|c|}
\hline Figure & $\begin{array}{c}E \\
\mathrm{~V} / \mathrm{cm}\end{array}$ & $A_{1}$ & $A_{2}$ & $\begin{array}{c}t_{1} \\
\text { min }\end{array}$ & $\begin{array}{c}V_{\text {av }} \\
\mathrm{cm} / \mathrm{min}\end{array}$ & $\begin{array}{c}t_{2} \\
\text { min }\end{array}$ & $\begin{array}{l}d_{1} \\
\mathrm{~cm}\end{array}$ & $\begin{array}{c}t_{3} \\
\text { min }\end{array}$ & $\begin{array}{c}v_{P L} \\
\mathrm{~cm} / \mathrm{min}\end{array}$ & $R \varphi=1$ & $R \varphi=0.80$ & $\begin{array}{c}K_{d} \varphi=1 \\
\mathrm{nM}\end{array}$ & $\begin{array}{c}K_{d \varphi}=0.80 \\
\mathrm{nM}\end{array}$ \\
\hline S5-a, 1 & & $4.44 \times 10^{5}$ & $8.17 \times 10^{5}$ & 2.89 & 3.34 & 0.892 & 2.98 & 3.54 & 0.842 & 0.536 & 0.480 & 0.470 & 0.366 \\
\hline S5-a, 2 & 200 & $4.57 \times 10^{5}$ & $9.07 \times 10^{5}$ & 2.82 & 3.43 & 0.967 & 3.32 & 3.99 & 0.831 & 0.519 & 0.464 & 0.437 & 0.340 \\
\hline S5-a, 3 & & $4.79 \times 10^{5}$ & $8.78 \times 10^{5}$ & 2.85 & 3.38 & 0.929 & 3.14 & 3.74 & 0.840 & 0.545 & 0.489 & 0.489 & 0.381 \\
\hline S5-b, 1 & & $4.71 \times 10^{5}$ & $1.21 \times 10^{6}$ & 2.87 & 3.37 & 0.917 & 3.09 & 6.31 & 0.489 & 0.626 & 0.573 & 0.713 & 0.556 \\
\hline S5-b, 2 & 120 & $4.82 \times 10^{5}$ & $1.28 \times 10^{6}$ & 2.83 & 3.41 & 0.950 & 3.24 & 5.93 & 0.546 & 0.574 & 0.519 & 0.559 & 0.435 \\
\hline S5-b, 3 & & $4.64 \times 10^{5}$ & $1.15 \times 10^{6}$ & 2.81 & 3.43 & 0.971 & 3.33 & 6.50 & 0.513 & 0.630 & 0.577 & 0.726 & 0.566 \\
\hline
\end{tabular}

See the accompanying Excel file for full data and error calculations 


\section{Note 7: Determination of $v_{\mathrm{L}}$}

The value of $v_{\mathrm{L}}$ was determined by electrophoretically running a plug of pure L (MutS aptamer) with a cathode at the inlet using the following conditions: $d=4.4 \mathrm{~cm}, r=37.5 \mu \mathrm{m}, l=1.09 \mathrm{~cm}$, pressure equal to $0.30 \mathrm{psi}$, total capillary length $=50 \mathrm{~cm}, E=200 \mathrm{~V} / \mathrm{cm}$ (Figure S6). To achieve faster detection time of pure L under standard IFCE condition, the sample plug was propagated by injecting a $5.8 \mathrm{~cm}$ long plug of the run buffer with a pressure pulse of $0.3 \mathrm{psi} \times 90 \mathrm{~s}$, resulting in a shorter $d(4.4 \mathrm{~cm})$. The value of $t_{4}$ was found directly from the electropherogram in

Figure S6: $t_{4}=714 \mathrm{~s}$. The, the value of $v_{\mathrm{L}}=0.006 \mathrm{~cm} / \mathrm{s}$ was found with eq 21 in the main text.

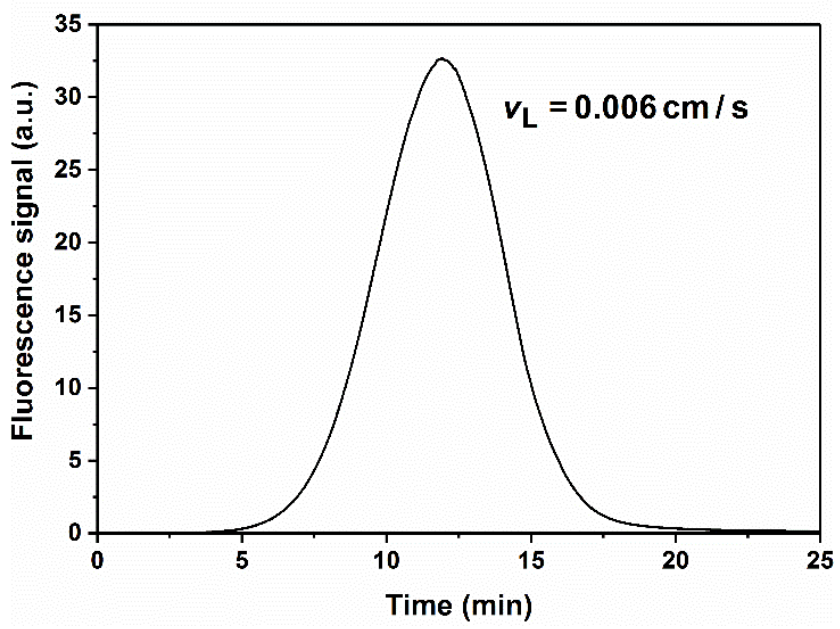

Figure S6. Determination of $v_{\mathrm{L}}$. Concentration of L (MutS aptamer) was $50 \mathrm{nM}$. 